\title{
Influences of individual-related factors and job satisfaction on workplace deviant behaviour among support personnel in Malaysian public service organizations.
}

\begin{abstract}
Workplace deviance has become pervasive in most organizations today. This cross-sectional study examines the influences of individual-related factors and job satisfaction on workplace deviance behaviour among 429 support personnel in Malaysian public service organizations. Samples were randomly selected using multi-stage cluster sampling. The findings of the study indicated that agreeableness, negative affectivity, conscientiousness, emotional intelligence and job satisfaction predicted the organizational deviant behaviour. The same variables, except for emotional intelligence, also correlated to interpersonal deviant behaviour. Implications and suggestion for future research are discussed.
\end{abstract}

Keyword: Workplace deviant behaviour; Job satisfaction; Negative affectivity; Conscientiousness; Agreeableness; Emotional intelligence. 